\title{
Marguerite d'Anjou,
}

\section{du monstre à la victime}

\section{Rewriting History: Margaret of Anjou, from Monster to Victim}

\author{
FLORENCE FIX
}

\section{Université de Rouen}

Marguerite d'Anjou, Française devenue reine d'Angleterre, fut une femme puissante du moyen âge, qui mena bataille pour défendre la couronne de son fils unique. Shakespeare la qualifie

Mots-clés

Marguerite d'Anjou ; Pixerécourt ; La Harpe ; mélodrame ; guerres civiles. de sorcière ayant contribué à la guerre civile britannique, la guerre des Deux-Roses. Les réécritures françaises de ce personnage historique deux siècles plus tard modifient complètement ce portrait critique. Jolie femme qui aime la séduction et le pouvoir, chez l'Abbé Prévost, reine digne de respect chez le tragédien La Harpe, elle est une malheureuse victime chez Pixerécourt et Meyerbeer.

L'interprétation dépend du registre adopté (roman, tragédie, mélodrame) dans lequel se lisent les préoccupations du temps. La représentation des femmes et la conception du pouvoir à l'époque de la réécriture y jouent un rôle majeur.

Margaret of Anjou was a powerful $15^{\text {th }}$ century woman; poor and landless, the French-born princess became Queen of

Keywords

Margaret of

Anjou;

Pixerécourt; La

Harpe;

mélodrame; civil wars.
England by marrying Henry VI. Since the King was prone to attacks of dementia, she took up arms to defend her only son's right to the throne. Shakespeare sees her as a sorceress, holding her responsible for the civil war. Rewriting her story two centuries later, French authors tend to rewrite history as well. She is a pretty seductress, an adventurer who loves power, according to Abbé Prévost. She embodies an illegitimate queen although worthy of respect in La Harpe's tragedy. As for the composers of melodramas and operas Pixerécourt and Meyerbeer, they depict her as an unfortunate victim. Interpretation depends on whether the text is a novel, a tragedy or a melodrama. It depends too on how women and power are seen in the writing context.

«Le courage extraordinaire de Marguerite, ses succès, ses malheurs, son caractère toujours supérieur à sa fortune, sont faits pour intéresser, soit dans une histoire, soit dans une tragédie, soit dans un mélodrame » (Pixerécourt, 1971 : 586). Cette Marguerite que le critique théâtral du Journal des débats, Julien Louis Geoffroy, présente en 1810 en figure toute destinée à la réécriture, adaptable à toutes les registres, ce n'est pas Marguerite de Valois, la célèbre reine Margot immortalisée par Alexandre Dumas, mais Marguerite d'Anjou, nièce du roi français Charles VII, dont le mariage avec Henri VI d'Angleterre au milieu du XVe siècle, était censé mettre fin à la guerre de cent ans entre la France et l'Angleterre. Dépeinte par Shakespeare au XVI siècle comme une 
nouvelle Hélène de Troie, n'ayant apporté que la discorde entre les deux pays, elle revient en France aux XVIII et XIX e siècles, «tranquille en son adversité » (La Harpe, 1814 : 1), dans des pièces qui célèbrent «la courageuse Marguerite» (Pixerécourt, 1971: 594), injustement persécutée par les Anglais. La réinterprétation d'une figure historico-littéraire du Moyen Âge à la modernité nous invite ainsi à aborder la réécriture sous l'angle de la redistribution de ses enjeux politiques. S'agissant d'un personnage de reine combattante, les questions d'autorité, d'exemplarité et de représentativité sont essentielles: la réécriture répond à un impératif d'explication et d'actualisation inféodé aux débats du présent sur le pouvoir. Elle implique l'infléchissement d'une figure historique (ici, une reine considérée en son temps comme une usurpatrice) vers la représentation qu'en a l'époque qui s'en empare (une victime, une femme malheureuse) et y superpose une symbolisation idéologique.

\section{Falsification de l'Histoire}

Réécrire l'histoire de Marguerite, princesse française et reine d'Angleterre du XVe siècle, c'est réécrire l'Histoire des deux pays en adoptant un point de vue et en défendant une interprétation du conflit. Il en va d'enjeux nationalistes et patriotiques : jeune fille ambitieuse, dans la première partie d'Henri VI (1592) de Shakespeare, elle prend violemment les armes à la place de son faible époux afin de défendre le droit au trône de leur fils unique. Dans un cycle de pièces écrites à la gloire d'un Royaume-Uni enfin apaisé après des décennies de guerre, les Français sont qualifiés de «nécromants et sorciers », Jeanne d'Arc de "sorcière de malheur » et de «nouvelle Circé». Dans le même ordre d'idées, le personnage nommé Margaret est une Française sans le sou, fourbe et adultère. Rien d'étonnant à ce que Marguerite rajeunisse et s'assagisse nettement dans les tragédies et mélodrames français deux siècles plus tard. « Reine cruelle », «femme barbare » (La Harpe, 1814 : 66), dont les Anglais reconnaissent toutefois la bravoure au combat dans la tragédie, tandis qu'il s'agit rien de moins que d'« exterminer ces farouches insulaires » (Pixerecourt, 1971: 628) dans le mélodrame qui la consacre en victime, Marguerite gagne en talents, en séduction et en qualités en revenant sous forme fictionnelle en France.

Le processus de réécriture et de littérarisation passe d'un côté à l'autre de la Manche par un écrivain dont on oublie parfois aujourd'hui qu'il fut un ardent vulgarisateur de la culture britannique en France : l'abbé Prévost, auteur de Manon Lescaut (1731), que Louis-Sébastien Mercier qualifie de « copiste éternel», qui « ignore la langue de Milton et de Shakespeare, et qui ne sait pas même la sienne» (Mercier, 1801: xv), prétend s'appuyer uniquement sur des sources anglaises pour rédiger son Histoire de la reine Marguerite (1740). Il diffuse en France le portrait d'une femme sans fortune mais si belle qu'elle est convoitée par le roi d'Angleterre, par son émissaire, Suffolk, et par son neveu et rival, le Duc de Glocester. D’Hélène de Troie la maléfique, la reine glisse vers Iseult la passionnée. Si le romancier reste en cela captif d'une représentation de Marguerite comme métaphore, voire source, de la guerre civile en Angleterre, il contribue d'ores et déjà à installer en France l'image d'une femme injustement malmenée.

L'abbé Prévost produit une Histoire en quatre volumes relatant la vie de Marguerite de son mariage à son décès, non sans approximations ni digressions nombreuses. La Harpe et Pixerécourt opèrent une réécriture trans-générique, passant du long texte en prose documenté à la pièce de théâtre. Le premier rédige une tragédie jouée au Théâtre-Français en 1763, le second un mélodrame représenté au théâtre de la Gaîté en 1810. La transformation du matériau historique en matériau fictionnel implique un resserrement de l'action sur un moment de la vie du personnage, infléchissant l'histoire vers l'anecdote. En lieu et place d'une trajectoire 
politique longue, faite d'intrigues, d'alliances, de revirements, une tension agonistique concentrée sur quelques jours de la vie d'une jeune reine. Il en découle des arrangements avec les faits historiques. Afin que Marguerite apparaisse sur la scène du mélodrame comme une veuve éplorée, en 1462 lors d'une bataille décisive, Pixerécourt prétend son mari Henri mort poignardé et leur fils trop jeune pour défendre ses droits, alors que le roi fut assassiné, tout comme son fils, en 1471. «La supposition de sa mort devenant favorable à mon drame, j’ai cru pouvoir me permettre ce léger anachronisme» (Pixerécourt, 1971: 604), indique l'auteur en note. Pourtant il ne s'agit pas que d'anachronisme mais d'une interprétation, liée à la nécessité formelle du pathos: présenter Marguerite comme une veuve en péril revient à valider sa légitimité à la couronne. Le mélodrame prend clairement parti pour la reine et présente son adversaire comme un dangereux rebelle. Il invente à Marguerite un chef d'armée français épris d'elle et condamne Gloucester, son rival prétendant à la couronne, à mourir brûlé dans l'incendie qu'il avait préparé pour elle. L'Histoire est manipulée afin de répondre aux exigences du registre de réécriture : dans l'histoire que l'abbé Prévost lui consacre, Marguerite est une aventurière adroite, qui tire parti de l'indécision de son époux pour servir ses intérêts. Le roi d'Angleterre Henri VI, autre Des Grieux, se laisse mener par la belle ambitieuse française. Dans la tragédie, « un drame de la plus grande simplicité » (La Harpe, 1814 : vii) selon son auteur, elle est une veuve désolée qui ne participe guère aux intrigues politiques; toute "reine terrible» (36) qu'elle soit, aux yeux de ses adversaires, l'action se joue ailleurs, entre hommes, c'est-à-dire entre le fidèle Warwick et le roi Édouard qui le déçoit. Dans le mélodrame enfin, elle n’a d'yeux que pour son jeune fils, que ses ennemis veulent assassiner. Comme il se doit dans ce genre pathétique, les traitres périssent d'une façon spectaculaire, dans l'effondrement d'un pont ou l'embrasement d'une forêt, et l'innocence persécutée est sauvée par une intervention providentielle. Relecture approximative et souvent factuellement fautive du passé pour qu'il offre matériau fictionnel adaptable aux exigences romanesques ou théâtrales du présent, l'événement historique se plie ainsi à tous les compromis, jusqu'à l'erreur assumée. De la femme qui mena des armées, fut sans pitié envers ses ennemis capturés, le mélodrame fait une malheureuse digne de compassion. Conformément aux principes conservateurs qu'il défend, le mélodrame montre que la détresse est une épreuve utile, une «école de l'adversité » (Pixerécourt, 1971 : 604), propre à exalter les tempéraments et les vertus. La guerre de Cent Ans et la guerre des Deux-Roses ne forment plus alors qu'un arrière-plan confus dont le désordre sert à mettre en évidence la rigueur morale des protagonistes. "Ce sont les circonstances qui font les héros » (642): la formule est réversible, les héros font les circonstances, car le dramaturge crée de toutes pièces les conditions d'émergence de valeurs et de gestes hérö̈ques.

L'événement historique entre également en collision avec les préoccupations politiques du présent d'écriture. Les pièces de Pixerécourt et de La Harpe sont produites à cinquante ans l'une de l'autre: période pendant laquelle la France passe, avec la césure de la Révolution française, de la monarchie à la République, de la République à l'Empire. En outre, la guerre franco-anglaise (1778-1783) puis l'opposition du Royaume Uni aux aspirations napoléoniennes font du voisin Outre-Manche plus que jamais une «perfide Albion ». Aussi la perception et la représentation de la guerre civile anglaise du XVI ${ }^{\mathrm{e}}$ siècle font largement les frais de l'actualité du XIX ${ }^{\mathrm{e}}$ siècle. Aux sources de ses malheurs, Marguerite dénonce «la coupable mobilité d'un parlement toujours prêt à se ranger du côté du plus fort» (Pixerécourt, 1971: 603) et l'inconstance du peuple, propos peu vraisemblables pour une souveraine anglaise médiévale, mais auquel le public du théâtre français de 1810, à l'apogée du Premier Empire, ne peut 
qu'être sensible. Le peuple chez La Harpe est instrumentalisé dans le discours de la reine comme une menace adressée à son rival. La versatilité de la populace pourrait l'amener en effet à servir ses intérêts contre le Duc :

\author{
Songe que dans ces murs un peuple factieux, \\ Toujours prêt à pousser un cri séditieux, \\ Cruel dans ses retours, extrême en ses offenses, \\ Peut encore à mon cœur préparer des vengeances, \\ Et m'offrir un plus sûr et plus facile appui, \\ Que ces Rois toujours lents à s'armer pour autrui. (1814:10)
}

La critique de La Harpe ne va pas contre les Anglais mais contre un pouvoir abusif: Marguerite et d'autres personnages de la pièce sont victimes de la légèreté du souverain. «L'Anglais environné du meurtre et des ravages» (13) est un peuple meurtri par l'ambition démesurée de la noblesse, qui après avoir mené un conflit d'un siècle contre la France, dérive en une guerre civile. L'auteur quelques années plus tard d'un Éloge de Charles V (1767) et d'un Éloge de Henri IV (1769) est partisan d'une monarchie éclairée et d'un pouvoir tempéré par la représentation parlementaire. Aussi noircit-il le portrait d'Édouard IV en monarque intransigeant: la tragédie montre qu'il trompe ses plus fidèles serviteurs. En revanche, Pixerécourt, qui écrit sous l'Empire alors que le Royaume-Uni est ennemi de Napoléon, valorise un pouvoir fort et critique l'ingratitude des Anglais envers la reine d'origine française. Le mélodrame célèbre une armée valeureuse entièrement dévouée, à l'instar des célèbres grognards. Napoléon, vainqueur d'Austerlitz, d'Iéna, de Friedland, et de Wagram ne connaît pas encore le déclin : les spectateurs français de 1810 voient ainsi sur la scène populaire qu'est le théâtre de la Gaité, spécialisé dans les mélodrames à grand spectacle et grands sentiments, exalter la loyauté d'une armée envers sa souveraine : «Tous marchent vers Londres pour vous y faire couronner aux acclamations universelles de l'armée et des nombreux sujets qui vous sont demeurés fidèles » (Pixerécourt, 1971 : 671). Aux spectateurs qui ont connu la Révolution française, le mélodrame offre « un catalogue de solutions consolatoires » (Eco, 1993: 20) dans lequel, comme l'indique un personnage à son adversaire, "tout le sang que tu auras versé retombera goutte à goutte sur ta tête coupable » (Pixerécourt, 1971 : 651). Quant au brigand qui vient en aide à une reine en lui disant «Vous avez daigné me fournir l'occasion de réparer mes torts envers la société » (655), il valorise la marginalité, pour peu que celle-ci soit temporaire et s'engage à revenir dans le giron de l'ordre.

La reine Marguerite, telle que représentée par le mélodrame français, incarne la raison d'État contre la rébellion, le respect des accords conclus, fussent-ils arbitraires, contre l'inattendu. Quoique son adversaire ait des raisons objectives de prétendre à la couronne, elle est dépeinte comme seule légitime et s'adresse à lui en ces termes, revendiquant la violence étatique : «Ton père était un rebelle, il mérite son sort; et tu me prouves aujourd'hui que je fus coupable envers l'État, en ne faisant point partager son supplice à ton frère et à toi » (Pixerécourt, 1971 : 668). Cet imaginaire qui veut que le Moyen âge soit un temps de cruauté et de chaos confirme la difficulté pour en faire une tragédie : celle de La Harpe ne porte d'ailleurs pas directement sur Marguerite, mais sur «l'âme grande et sensible de Warwick» (La Harpe, 1814 : ix), ce « héros infortuné » (Prévost, $1740: 6$ ) en butte à l'ingratitude des puissants. Celle 
de Louis de Cahusac ${ }^{1}$, également intitulée Le Comte de Warwick, tragédie rédigée vingt ans avant celle de La Harpe, en 1742, ne passa pas la première représentation et ne fut jamais publiée. Le rebelle, le proscrit qui font les belles heures du théâtre romantique ne sont pas encore bienvenus dans des pièces qui composent avec la censure d'État. Face à l'injustice du pouvoir, le loyal Warwick, qui se décrit comme «l'arbitre, la terreur et le soutien des rois » (La Harpe, 1814 : 16), choisit le sacrifice plutôt que la contestation et accepte la mort. L'éloge du «peuple généreux» ou «peuple citoyen» qu'est la nation britannique a des accents d'exaltation parlementariste que l'on ne retrouve évidemment plus sous la plume de Pixerécourt, plus conservateur et plus circonspect quant aux aspirations à contrecarrer un pouvoir fort. Dans la tragédie, Marguerite se trouve en retrait, personnage secondaire émargeant parmi les nombreuses preuves du désordre du roi. Son ambition ne relève pas vraiment de l'bybris, elle est plutôt la marque du manque de consistance de son époux, incapable de contenir les ambitions des nobles et d'entendre les désirs du peuple, «Henri, dans la langueur, tombé presque en naissant/ Et d'une épouse altière esclave obéissant» (La Harpe, 1814 : 16). Égoïste comme tous les nobles chez La Harpe, elle n'a guère de singularité. Victime du pouvoir, elle est en revanche sur le devant de la scène émotionnelle que compose le mélodrame de Pixerécourt.

\section{Réécriture et emprunt}

Une anecdote mise en relief par le livre de l'abbé Prévost relate que la reine, perdue en forêt, impressionna tant par sa dignité et sa noblesse un bandit qu'il lui laissa, ainsi qu'à son fils, la vie sauve. Pixerécourt ne manque pas d'y consacrer tout un acte d'une pièce concentrée sur les émotions et les valeurs humaines. Réfugiée aux frontières de l'Ecosse, cachée dans une forêt encerclée par ses ennemis, Marguerite, comme toute héroïne de mélodrame, y rencontre l'inlassable péril de toute jeune femme vulnérable, le brigand. Les forêts des mélodrames sont en effet invariablement des espaces où urbanité et sauvagerie entrent en collision, offrant l'occasion de révéler la porosité des valeurs. Le brigand n'est pas un irrécupérable malfrat : il n'a été poussé à la marginalité que par l'injustice dont il a été victime : "Cet acte d'iniquité m'inspira la haine la plus violente, et me fit embrasser le vil métier que j'exerce. À ce titre, je dois te hair et me venger. Mais j'oublie tout en te voyant malheureuse ; dispose de moi et de ceux qui m'accompagnent» (1971: 641). Dans l'idéologie fortement moralisatrice du mélodrame, la faute est pardonnable dès lors qu'elle n'est qu'égarement temporaire dont la responsabilité est dirigée vers un tiers antérieur à l'intrigue. Poussé au crime par le spectacle de l'injustice, le bandit revient à la vertu par le spectacle de la dignité. Un roi méchant l'a jeté dans la forêt, une reine malheureuse l'en fait sortir. À l'issue de la pièce, tous se dirigent vers Londres afin de recouvrer leurs droits.

Le mélodrame a pour idéal le bonheur domestique : aussi l'exilé, le voyageur ou le proscrit sont-ils des figures victimaires, qui n'aspirent qu'à revenir au logis. À peine sort-on de chez soi qu'on est la proie de toutes les catastrophes. Une Marguerite esseulée, épuisée, portant son enfant dans ses bras, s'inscrit dans la longue liste de distribution des rôles théâtraux féminins qu'un jaloux, un espion ou un traitre condamnent à une détresse édifiante. Des actrices de renom au XIX ${ }^{\mathrm{e}}$ siècle se spécialisent dans ce registre lacrymal et «l'emploi des princesses innocentes, malheureuses et persécutées » est alors très disputé, selon un critique ironique, par «une foule de prétendantes aux honneurs du martyre mélodramatique » (Castel, 1829: 204).

\footnotetext{
${ }^{1}$ Cahusac est davantage connu pour avoir été le librettiste de Jean-Philippe Rameau que pour son activité d'auteur dramatique.
} 
Dans Marguerite d'Anjou, Mlle Bourgeois, «actrice qui dans sa jeunesse, faisait le coup de sabre comme le coup de poing, et qui a tué plus d'hommes en sa vie que le plus intrépide de nos grenadiers » (204) campe une mère inquiète qui a besoin d'une aide masculine pour se défendre. Le motif inspire des peintres néoclassiques comme François Dubois, auteur d'un Marguerite d'Anjou prise par les brigands (1832) ou Eugène Devéria avec un Marguerite d'Anjou, reine d'Angleterre, protégeant son fils Édouard, prince de Galles, pendant la guerre civile. La reine est dépeinte dans une posture passive et protectrice, qui occulte totalement la part active que le personnage historique a prise aux conflits.

La perte en forêt constitue l'un des passages obligés de la détresse féminine, très identifiables par les spectateurs qui s'attendent à tout moment à ce qu'un personnage de femme vulnérable soit sauvé de la noyade, de l'incendie, de l'assassinat par un inconnu galant homme. La rencontre avec le farouche brigand donne raison à l'inquiétude générée par le lieu sauvage mais l'invalide immédiatement par le recours aux valeurs de l'entraide et du respect. Chez Pixerécourt, le brigand, qui s'éprend de Marguerite au premier coup d'œil et s'offre à la servir, se nomme Carl, clin d'œil qui ne peut manquer de flatter le public d'un spectacle populaire se piquant de culture savante. La pièce de Schiller Les Brigands (Die Raüber), créée en Allemagne en 1782, avait eu un retentissement considérable en France dès l'année suivante et avait ouvert la voie à une série de brigands de théâtre au grand cœur et aux idées républicaines. L'opposition des frères ennemis, Karl et Franz Moor, dont l'un est un brigand et l'autre un usurpateur, consacre la floraison de drames portant sur la légitimité individuelle. Variation d'Abel et Caïn, Karl et Franz se disputent l'héritage paternel : le fils répudié, devenu brigand, se révèle porteur de valeurs humaines fortes, plus importantes que les valeurs financières et nobiliaires dont se réclame son cadet. La réécriture mélodramatique que donne Pixerécourt de la vie de la reine Marguerite entrelace les sources : à l'abbé Prévost, il emprunte la rencontre entre la reine et le brigand, qui ne se trouve pas chez Shakespeare, à Schiller le prénom du bandit par dépit. Il en résulte un maillage entre culture populaire et culture savante, jeux comiques et exaltation morale. Carl, le brigand amoureux, s'allie à un médecin gascon, personnage comique, pour sauver une reine d'Angleterre née princesse de Lorraine et de Bar. Cette combinaison de références produit également un effet d'écho, de redoublement des enjeux agonistiques : Carl comme Marguerite sont victimes d'usurpateurs ; l'un comme l'autre ne sont considérés comme des marginaux que par des envieux désireux de ternir leur image. Le mélodrame invite le spectateur à se méfier des apparences : la femme perdue en forêt, le bandit menteur ont au fond un cœur d'or. En revanche, le duc lourdement armé, au verbe haut, n'est pas fiable. Sa splendeur manifeste doit être comprise comme un artifice et une malhonnêteté. Le mélodrame est coutumier de cette structuration en miroir qui offre à ses spectateurs la satisfaction d'une lecture consolatrice. Il en va de même du traitre, invariable comparse du pouvoir abusif. Dans la pièce de Pixerécourt, il répond, comme Carl, à un nom de consonance allemande, Stoffel. Il n'est pas vraisemblable qu'un tel personnage soit allié aux York contre les Lancastre dans une forêt du Moyen âge anglais, mais pour un mélodrame de 1810, il fait pleinement l'affaire. La germanophobie est alors encouragée par le régime impérial qui a ravivé la suspicion forgée lors de la Révolution Française contre le voisin outre-Rhin susceptible d'œuvrer au retour de la monarchie. Aussi les traittres de mélodrame ont souvent des noms à consonance allemande. Dans La Femme à deux maris, grand succès de Pixerécourt en 1802, une femme est affligée d'un premier mari traitre à la patrie et violent envers elle, nommé Isidor Fritz, quand son second époux, vertueux colonel au service de l'Empereur, porte un nom indiquant la loyauté sacrificielle, Édouard, comte de Fersen. Patriotique et nationaliste, le mélodrame n'en a pas 
moins une conception universaliste des valeurs humaines et des émotions qui autorise les schémas actantiels les plus improbables historiquement. Des personnages de médecin gascon, de brigands écossais et de mercenaires français peuvent s'allier, pour peu qu'ils soient tous épris de la même femme, contre un noble britannique et un traitre allemand.

En effet, en réécrivant, un écrivain tisse son objet de ses propres croyances autant que de ses lectures et influences. Rédigeant une tragédie qu’il aspire à voir représenter à la ComédieFrançaise, La Harpe s'inscrit dans le sillage des œuvres qu'impliquent ce registre et ce lieu. Réflexion sur l'abus de pouvoir, l'ingratitude ou la loyauté des souverains, Le comte de Warwick a des accents cornéliens. Lorsqu'Élisabeth supplie le roi de se montrer magnanime envers Warwick qui a changé de camp en ces termes «Osez lui pardonner. Pour punir une offense,/ La générosité peut plus que la vengeance » (La Harpe, 1814 : 41), on croirait entendre Livie face à Auguste, dans Cinna de Corneille. Dénonçant un pouvoir abusif et offrant l'éloge d'un peuple aspirant à la liberté, la pièce ressemble aux tragédies d'inspiration romaine qui puisent parmi les souverains injustes comme Néron ou les souverains convertis à la clémence comme Auguste matière à réflexion sur la nécessité d'un pouvoir équilibré, capable de «le punir à force de bienfaits » (59).

L'Anglais, indépendant et libre autant que brave,

Des caprices de cour ne fut jamais esclave.

Nous ne l'avons point vu régler jusqu'à ce jour

Sur la faveur des rois sa haine ou son amour.

Contre un tel préjugé son âme est aguerrie ;

Souvent contre le trône il défend la patrie » (30),

écrit le tragédien. Le roi voit le pouvoir comme un fardeau et désavoue une alliance avantageuse pour son pays en faveur d'une passion amoureuse : il refuse d'épouser la sœur du roi de France car il est épris d'une veuve anglaise désargentée. Dans la tragédie, les égarements sentimentaux du roi sont la marque d'un pouvoir immérité et contestable. Dans le mélodrame, ce sont ces mêmes émotions qui garantissent la légitimité du pouvoir : Marguerite mère et épouse a tous les droits face à ses rivaux. En d'autres termes, le registre littéraire adopté tragédie ou mélodrame -, encastré dans un régime de croyance - confiance en la monarchie parlementaire éclairée ou valorisation d'un pouvoir impérial fort - détermine une interprétation idéologiquement appuyée des personnages historiques. Condensé d'histoire européenne manipulée, condensé d'histoire littéraire aussi, parfois jusqu'au pastiche involontaire, la réécriture engage une représentation du pouvoir.

\section{Femmes de pouvoir}

Le personnage historique de Marguerite, épouse française d'un roi britannique soumis à des crises de démence, prit à plusieurs reprises les armes contre le duc d'York qui menaçait de s'emparer du pouvoir royal. Elle défendit sur le champ de bataille les droits de succession de son fils unique, considéré par ses adversaires comme illégitime, et libéra son époux emprisonné. Petite-fille de Yolande d'Aragon, femme d'intrigues et de pouvoir très indépendante, qui assura le prestige de la maison d'Anjou tantôt à l'encontre des intérêts du roi de France, tantôt en alliance avec lui, c'était une femme de la fin du Moyen âge, guerrière et stratège. Fondatrice du Queens College de Cambridge en 1448, menant guerres et intrigues, elle apparaît même, chez Shakespeare, capable de tuer un enfant et de se moquer du père en deuil. 
Modèle d'intransigeance et de ténacité dont se souvient le romancier Walter Scott dans Anne of Geierstein or The Maiden of the Mist, que la traduction française retient couramment sous le titre de Charles le Téméraire en 1829, lorsqu'il la dépeint en forte femme, elle prête inévitablement à une interprétation genrée. Son opiniâtreté et sa bravoure la font reconnaitre aujourd'hui comme une vaillante combattante. Une adaptation télévisée récente de la Henriade (The Hollow Crown, Sam Mendes, 2012) met particulièrement l'accent sur la cruauté et la détermination d'une Margaret montrée comme une femme en armure tuant à l'arme blanche ses adversaires et faisant placer leurs têtes sur des piques. Mais la lecture romantique modifie la revendication en consternation, l'intrigue politique en intrigue sentimentale. Chez l'abbé Prévost, c'est une coquette qui joue des hommes pour conserver le pouvoir ; chez Pixerécourt, c'est une mère qui convainc des hommes d'assurer le pouvoir à son fils. Il n'est jamais question d'elle en tant que guerrière, stratège ou femme politique.

Il est utile à cet égard de considérer les autres personnages féminins utilisés dans les intrigues ainsi réécrites. Marguerite s'inscrit chez l'abbé Prévost dans une constellation de femmes de cour ambitieuses qui ne sont pas sans ressemblances avec la célèbre Manon Lescaut du même auteur ; la reine n'est pas la seule femme de l'Histoire à être «aussi dangereuse par la beauté que par les artifices» $(1740: 96)$ et le traducteur de Pamela ou la vertu récompensée de Samuel Richardson se plait à composer des portraits de femmes qui, comme Pamela, usent de leurs charmes pour réussir. Anne Neville, Eleonor Cobham, personnages historiques, sont de jolies femmes intrigantes et irrésistibles. En revanche, chez Pixerécourt, le personnel dramatique féminin se trouve réduit à deux figures : le chef de guerre épris de Marguerite est marié à une femme fidèle qui le rejoint, travestie en jeune soldat, sur le champ de bataille. Nécessaire à la composition finale et chorale qui clôt tout mélodrame, la loyale épouse offre l'occasion à la reine de s'effacer devant tant de dévouement et à l'époux de reconnaitre publiquement le triomphe de la vertu. La scène finale de l'opéra qu'en tire Meyerbeer en 1820, Margherita d'Anjou, fut un triomphe à la Scala de Milan. Enfin La Harpe, lecteur de Shakespeare et de Racine, isole Marguerite comme émouvante figure de la veuve prisonnière, soucieuse de la survie de son unique enfant. Avec l'hypotypose «Votre fils, cet objet de vos soins, de vos pleurs,/ Traine, loin des regards d'une mère avilie,/ Sous les yeux des tyrans son enfance asservie » (1814: 2), le tragédien s'essaye manifestement à suivre l'exemple d'Andromaque (1667) ou de Britannicus (1669) de Racine qui sont encore un siècle plus tard les modèles du genre. Le roi rival de Marguerite et de son époux, nouveau Néron, est un «astre sanglant»(13) inconstant et dangereux. Warwick, qui fait les frais de l'aveuglement politique du souverain, donne à sa propre épouse Marguerite en exemple. Alors que sa compagne propose de supplier le roi en sa faveur, il lui conseille plutôt de prendre les armes : «Égalez son courage, osez braver un roi» (52). Ennemi de la reine, il n'en éprouve pas moins du respect pour elle et reconnait son ardeur au combat. Cela ne signifie pas pour autant que la pièce ait des accents proto-féministes : il revient à une femme, l'épouse de Warwick, de considérer le ralliement à Marguerite comme une aberration: " $\grave{A}$ servir une femme il est donc descendu!/ Tu l'emportes sur nous, trop cruelle ennemie !/ Je cède en gémissant à ton fatal génie./ Il est de ton destin d'accabler mon pays » (60). Femme fatale, Marguerite l'est, soit en tant que stratège manipulatrice (dans la tragédie), soit en tant que veuve séduisante (dans le mélodrame) : son désir de pouvoir est toujours entaché de stéréotypes.

Ainsi, en faire une jolie femme dont la beauté renversante pousse invinciblement des soldats français et des brigands écossais à défendre ses intérêts n'est pas seulement un contresens historique. C’est une réappropriation du passé par le présent. «La plus malheureuse 
des reines, des épouses et des reines ", comme l'indique une plaque commémorative du château de Souzay à Dampierre sur Loire, est une création du XIX ${ }^{\mathrm{e}}$ siècle. Si ce n'est pas dans ce manoir, restauré dans le style néogothique au XIX ${ }^{\mathrm{e}}$ siècle que la reine exilée mourut en 1482, mais dans un château voisin, la construction de son image passe aussi par ce geste de redistribution du patrimoine monumental. La légende de Marguerite d'Anjou s'inscrit, quatre cents ans après sa mort, dans la célébration d'une coquette rassemblant autour d'elle « tous les hommes braves, déterminés et amants de la beauté » (Pixerécourt, 1971 : 596). Il n'en va pas seulement d'une représentation du féminin, mais de la nation française, les Français « défenseur du beau sexe» (604). Écartant le fait que les guerres médiévales furent surtout l'affaire de mercenaires, l'auteur de mélodrame présente les conflits comme des engagements consentis afin d'aider la veuve et l'orphelin. De la conscription obligatoire, il n'est pas davantage question: des hommes braves volent spontanément au secours d'une femme vertueuse en danger, des brigands à sa vue se convertissent à l'honnêteté et à la loyauté. Dépeinte comme une épouse éplorée d'un roi assassiné, en passant sous silence les désaccords des époux et la démence du roi, protégeant un enfant vulnérable, Marguerite reine médiévale devient une ménagère inquiète, « mère infortunée » (624) à la dimension quasi mariale.

Son destin toutefois ne diffère pas de celui de nombreuses aristocrates européennes de son temps, qui prirent les armes, exercèrent des régences pour leurs fils au berceau et intriguèrent afin de conserver le pouvoir. En faire une victime, une mère inquiète, désireuse de protéger son fils, éprise d'un époux emprisonné, relève d'une conception beaucoup plus tardive de la maternité et de la féminité. La Marguerite de mélodrame n'envisage jamais le pouvoir en soi, mais uniquement par délégation : si elle lève une armée, c'est parce que son époux ne peut plus le faire, et parce que son fils ne le peut pas encore. Comme Roxane au siège d'Arras dans le Cyrano de Bergerac d'Edmond Rostand, Marguerite enflamme les troupes à se battre et s'offre en récompense au chef d'armée. "Si je recouvrais ma couronne et qu'il me fût permis de la partager avec vous, je croirais ne pouvoir mieux servir ma nation qu'en m'associant un héros qui mettrait son bonheur et sa gloire à défendre mes droits » (611). Veuve dans la pièce de Pixerécourt, elle peut certes envisager un remariage : il est toutefois peu probable qu'une fois revenue sur le trône britannique, Margaret aurait fait l'erreur politique d'épouser un chef de guerre français.

L'abbé Prévost rappelle à ses lecteurs, afin de justifier le comportement de Marguerite d'Anjou, « qu'on était dans un siècle où les fameux exemples de Jacqueline de Brabant, et d'une Princesse de France, Reine Douairière d'Angleterre, avaient mis la galanterie fort en honneur. L'esprit et la beauté étaient le seul mérite qu'on cherchait dans les femmes, et c'en était encore un plus grand pour elles de s'être distinguées par des aventures éclatantes, qui servaient comme de lustre à la perfection de leur caractère » $(1740: 121)$. En prétendant que telles étaient les mœurs au Moyen âge, l'abbé s'offre la possibilité de multiplier les allusions grivoises, les récits à rebondissements de mariages et d'adultères tout en prétendant ne pas les cautionner. Simplifiant la géopolitique, il subordonne les enjeux territoriaux qui animent les conflits de l'époque à des motivations sentimentales. Marguerite est jalouse, amoureuse, soucieuse de favoriser financièrement et socialement ses amants. Les autres femmes de la cour ne sont pas en reste: tentatives d'assassinat, empoisonnement d'un rival, vengeance, noyade et changements rapides d'amant sont leur occupation durant les quatre volumes composés par l'abbé. Les complexités des rivalités nobiliaires et des successions dynastiques sont passées sous silence, au profit de multiples péripéties motivées par la séduction ou la vengeance. En 1810 en revanche, le retour de l'ordre moral voulu par l'idéologie napoléonienne interdit de penser 
l'autonomie combattive de la reine Marguerite comme une volonté de pouvoir. C'est bien un enjeu éthique et politique, autant qu'esthétique qui amène une réécriture mélodramatique à présenter une reine déchue en victime désolée: si dans la tragédie de La Harpe, la reine étrangère à la légitimité controversée est admirée et respectée par le roi Édouard IV qui reconnaît en sa rivale une interlocutrice digne, en revanche dans le mélodrame la reine est insultée par un Duc de Gloucester rebelle à toute autorité, instrument de désordre et de désunion. Image de la maternité souffrante, elle n’aspire à la couronne que pour la poser sur la tête de son fils unique. L'insulte fait partie des nombreuses avanies qu'elle subit avec abnégation pour y parvenir.

Les variations successives autour d'une figure historique ressortissent d'un «syndrome narratif» en ce que «Ces narrations imbriquées nous fabriquent une mémoire qui structure notre identité, notre sensibilité et détermine notre rapport au monde» (Farrugia, 2008: 83). Ainsi la reine du $\mathrm{XV}^{\mathrm{e}}$ siècle britannique est chez Pixerécourt une femme française qui respecte le Code civil établi par Napoléon en 1804 : elle ne s'enhardit à réclamer des droits en faveur de son fils que parce qu'elle est veuve. Encore lui faut-il dans cette entreprise s'assurer par sa jolie figure le soutien de soldats et de brigands amoureux. Chez La Harpe, elle est une reine respectée mais cruelle à l'instar de l'Impératrice Catherine de Russie, couronnée un an avant la parution de la pièce en 1762 après avoir, comme Marguerite, donné naissance à un enfant unique à la filiation douteuse et vu mourir son fragile époux en prison. La réécriture du personnage historico-littéraire est arrimée à une interprétation qui ne tient pas tant aux faits avérés qu’à la conception de la condition féminine lors de la rédaction des pièces.

Entre également en jeu une conception du pouvoir: le comportement de Marguerite, brutale et égoïste, préoccupée par la conservation des privilèges nobiliaires, s'offre en contrepoint et en contre-exemple du dévouement de Warwick dans la tragédie de La Harpe. Inversement chez Pixerécourt, c'est elle qui est donnée en modèle de loyauté et de bravoure. En empruntant à Shakespeare un personnage peu connu de l'Histoire franco-britannique, les auteurs proposent aux spectateurs un jeu sur la proximité et la distance, somme toute emblématique du processus même de la réécriture. Distance, parce que Marguerite, fille du roi René, prise dans les complexités de la guerre des Deux-Roses, n’a pas la notoriété de grandes figures des guerres civiles françaises, notamment des guerres de religion qui fascinent les romantiques. Proximité néanmoins aussi, car les enjeux politiques que sa trajectoire improbable de femme sans fortune à souveraine en armure soulèvent restent prégnants pour des spectateurs du XIX siècle français. La question de la légitimité de Marguerite est fondamentale; celle d'une réécriture envers ses sources l'est aussi. C'est précisément dans le tissage établi entre l'Histoire et la fiction que joue pleinement la fonction réflexive de la reprise.

\section{BIBLIOGRAPHIE :}

LA HARPE, Jean-François de (1814). Le Comte de Warwick, tragédie en 5 actes et en vers (1763). Brest : Imprimerie de Michel.

PIXERÉCOURT, René-Charles Guilbert de (1971). Marguerite d'Anjou (1810). In Théâtre choisi. Volume 2. Genève : Slatkine.

PRÉVOST, Antoine-François (1740). Histoire de Marguerite d'Anjou, reine d'Angleterre. Tome 1. Amsterdam : Desbordes.

SHAKESPEARE, William (1997). Henri vi parties 1, 2 et 3 (1588-1593). In Histoires ii. Paris : Robert Laffont. 
$* * *$

CASTEL, Louis (1829). Mémoires d'un claqueur. Paris : Constant-Chantpie.

ECO, Umberto (1993). De Superman au surhomme (1978). Paris : Le Livre de poche.

FARRUGIA, Francis et al. (2008). Émotions et sentiments : une construction sociale. Paris: L'Harmattan.

MERCIER, Louis-Sébastien (1801). Néologie, on Vocabulaire de mots nowveaux, à renouveler, on pris dans des acceptions nowvelles. Tome 1. Paris : Moussard et Maradan. 